\title{
Enfrentamento de Estresse no Trabalho: Relações entre Idade, Experiência, Autoeficácia e Agência
}

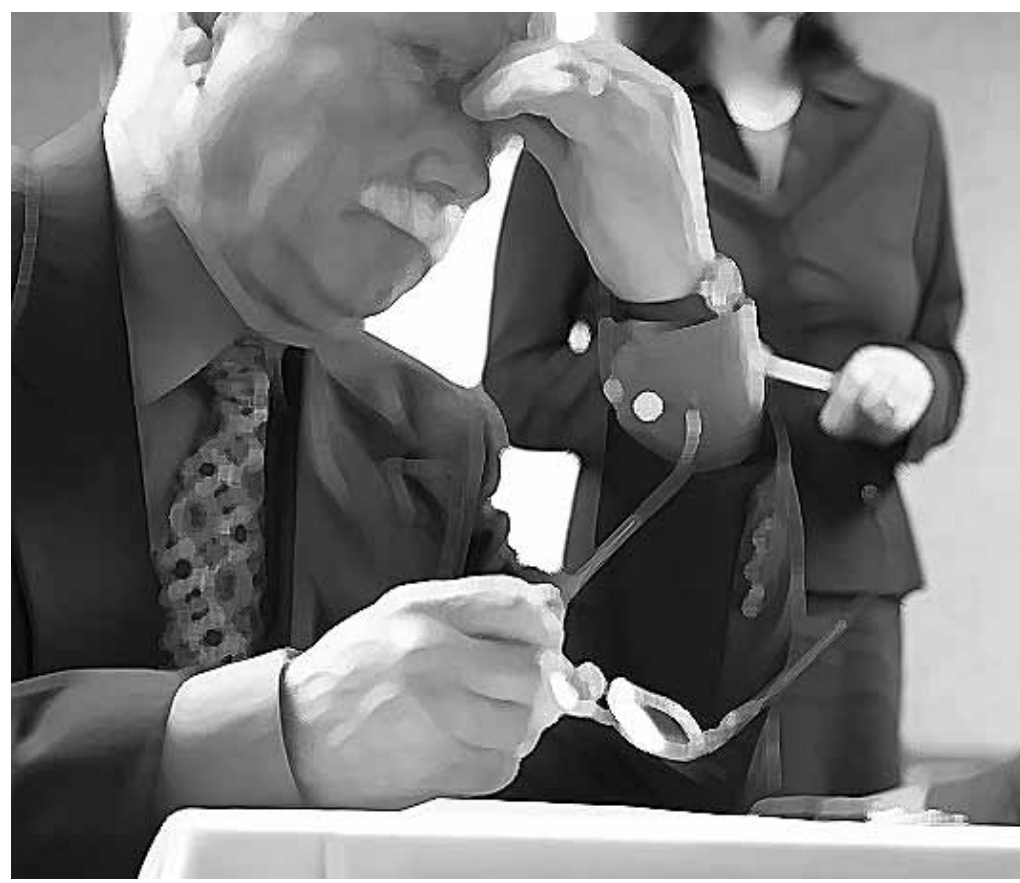


Resumo: Este trabalho constitui um estudo descritivo que investigou as relações entre as estratégias de enfrentamento de estresse no trabalho e o estresse percebido, a idade, o cargo e a experiência de trabalho, mediadas pelas crenças de autoeficácia e de agência, entre 71 líderes de uma companhia. Método: o trabalho incluiu um questionário de dados sociodemográficos, de escalas de avaliação do grau de estresse percebido, do enfrentamento no trabalho, de crenças de autoeficácia e de agência. $\mathrm{O}$ grau de estresse percebido foi 7(0-10). As estratégias de controle foram frequentemente utilizadas $(M=3,73 ; \mathrm{DP}=, 49)$, esquiva, algumas vezes $(M=2,07 ; D P=, 50)$, e manejo de sintomas, raramente $(M=1,86 ; D P=, 44)$. A autoeficácia foi moderada $(3,23, \mathrm{DP}=3,37)$. Foram encontradas correlações positivas entre experiência de trabalho e autoeficácia (,27), autoeficácia e controle (,53), crenças de agência pessoal e controle e autoeficácia (,67 e ,57, respectivamente). A análise de regressão logística multivariada mostrou que líderes com escores mais altos em estratégias de controle apresentaram escores mais altos em autoeficácia e agência. Conclusão: os resultados apontam o valor da experiência de trabalho, da autoeficácia e da agência para o envelhecimento e o enfrentamento dos estressores de trabalho, principalmente em tarefas que envolvem risco de vida, mostrando que as organizações deveriam valorizar sua mão de obra madura.

Palavras-chave: Estresse. Trabalho. Enfrentamento. Auto-eficácia.

Abstract: Objective: this is a descriptive work that investigated the relations between coping at work and perceived stress, age, job position, job experience, mediated by self-efficacy and agency beliefs, among 71 leaders of a company. Method: the work included a sociodemographic questionnaire, scales of perceived stress intensity, coping at work, self-efficacy and agency beliefs. Results: the degree of perceived stress was $7(0-10)$. Control strategies were frequently used $(M=3,73$ and $S D=, 49)$, avoidance, sometimes $(M=2,07$ and $S D=, 50)$ and management of symptoms, rarely $(M=1,86$ and $\mathrm{SD}=, 44)$. Self-efficacy was moderate $(3,23, \mathrm{SD}=3,37)$. Positive correlations were observed among job experience and self-efficacy (,27), self-efficacy and control (,53), personal agency beliefs and control and self-efficacy (,67 and ,57, respectively). The multivariate logistic regression analysis showed that leaders with the highest scores in control strategies had higher scores in self-efficacy and agency. Conclusion: the results highlighted the value of job experience, self-efficacy and agency beliefs for aging and coping at work, mainly in life threatening risk tasks, showing that the organizations should value mature employees.

Keywords: Stress. Work. Coping behavior. Self-efficacy.

Resumen: Este trabajo constituye un estudio descriptivo que investigó las relaciones entre las estrategias de enfrentamiento de estrés en el trabajo y el estrés percibido, la edad, el cargo y la experiencia de trabajo, mediadas por las creencias de auto-eficacia y de agencia, entre 71 líderes de una compañía. Método: el trabajo incluyó un cuestionario de datos socio-demográficos, de escalas de evaluación del grado de estrese percibido, del enfrentamiento en el trabajo, de creencias de auto-eficacia y de agencia. El grado de estrés percibido fue $7(0-10)$. Las estrategias de control fueron frecuentemente utilizadas $(M=3,73 ; \mathrm{DP}=, 49)$, esquiva, algunas veces $(M=2,07 ; D P=, 50)$, y manejo de síntomas, raramente $(M=1,86 ; D P=, 44)$. La auto-eficacia fue moderada $(3,23, \mathrm{DP}=3,37)$. Fueron encontradas correlaciones positivas entre experiencia de trabajo y auto-eficacia $(, 27)$, auto-eficacia y control $(, 53)$, creencias de agencia personal y control y auto-eficacia (,67 y, 57, respectivamente). El análisis de regresión logística multi-variada mostró que líderes con puntuaciones más altas en estrategias de control presentaron puntuaciones más altas en auto-eficacia y agencia. Conclusión: los resultados apuntan el valor de la experiencia de trabajo, de la auto-eficacia y de la agencia para el envejecimiento y el enfrentamiento de los estresores de trabajo, principalmente en tareas que envuelven riesgo de vida, mostrando que las organizaciones deberían valorizar su mano de obra madura.

Palabras clave: Estrés. Trabajo. Enfrentamiento. Autoeficacia.

A revisão de literatura acerca do enfrentamento do estresse sugere que ele é influenciado pela avaliação do contexto no qual a siuação estresssante ocorre (Folkman \& Mosckowitz, 2000; Lazarus, 1993). O modelo cognitivo de Lazarus reconhece que a avaliação cognitiva da situação tem uma função central na compreensão do enfrentamento. A avaliação é o processo que medeia, de um lado, as demandas, as limitações e os recursos do meio e, de outro, a hierarquia de objetivos e as crenças pessoais. Uma das mais importantes 
características do modelo é que o valor das estratégias de enfrentamento depende do tipo de situação enfrentada, da situação estudada (por exemplo, o bem-estar, a saúde física, o trabalho) e do tipo de personalidade (Lazarus, 1993).

No ambiente de trabalho, a avaliação cognitiva da situação estressante inclui o reconhecimento da ameaça, as estratégias de enfrentamento selecionadas e a percepção de si mesmo como alguém capaz ou eficaz para lidar com os estressores. Aqueles que são confiantes em suas habilidades para realizar tarefas podem usar mais efetivamente estratégias para lidar com os estressores (Bandura, 1997; Jex, Bliese, Buzzell, \& Primeau, 2001; Latack, 1986).

Algumas das características do trabalho podem dar lugar a desajustes, a sofrimento, a esgotamento e a doenças psiquiátricas; entre elas, podem ser citadas: sobrecarga quantitativa (pressão no tempo e fluxo de trabalho repetitivo) e qualitativa (falta de variação de estímulo e de oportunidade para o exercício da criatividade, solução de problemas e de interação social), conflitos de papéis no contexto do trabalho e entre papéis familiares e laborais, falta de controle sobre a situação de trabalho (o trabalhador não tem autonomia sobre seu ritmo e método de trabalho), falta de apoio social (envolvendo amigos e família), presença de estressores físicos (ex. ruídos, odores, luzes, temperatura, produtos químicos), uso de tecnologia de produção em série, processos de trabalho automatizados, riscos físicos e psicológicos e trabalho em turnos. Incluem-se ainda como estressores os conflitos interpessoais (Figueroa, Schufer, Muiños, Marro, \& Coria, 2001), além dos conflitos entre demandas familiares e do trabalho (Michie \& William, 2003).

Latack (1986) mensurou o enfrentamento do estresse no ambiente de trabalho considerando três tipos de estratégias: 1 . ações e reavaliações cognitivas de resolução de problemas (por exemplo, Falar com meu supervisor para discutir o problema), 2. ações e reavaliações cognitivas de esquiva (por exemplo, Tentar ficar fora das situações) e 3. manejo de sintomas, referindo-se às ações para manejo do estresse relacionado, por exemplo, à prática de exercícios físicos, de um esporte, à dança ou ao relaxamento.

\section{Enfrentamento e envelhecimento no trabalho}

Não há consenso na literatura sobre o fato de o enfrentamento mudar de acordo com a idade. Estudos com o Teste de Apercepção Temática mostraram que os idosos se tornam mais passivos, enquanto outros estudos revelaram que os esforços de enfrentamento aumentam com a idade, como o uso de mecanismos de defesa mais maduros, tais como o altruísmo (Aldwin \& Gilmer, 2003). Adultos mais velhos utilizam tanto estratégias centradas no problema quanto estratégias de fuga-esquiva (Aldwin, 1991; Aldwin \& Gilmer, 2003; Aldwin, Sutton, Chiara, \& Spiro III, 1996). Eles tendem a avaliar os problemas como menos estressantes, porque consideram-se menos responsáveis por eles, não havendo diferença entre os adultos mais jovens e os mais velhos. Apesar dos problemas e das perdas significativas relacionadas à idade, eles aprendem a diferenciar os estressores controláveis dos incontroláveis, sendo essa importante tarefa do desenvolvimento que contribui para a saúde mental dos mais velhos.

Um estudo conduzido com 634 adminstradores em Hong Kong mostrou que a relação entre a idade e os indicadores de bemestar foi modelada por uma combinação de estratégias de enfrentamento, lócus de controle percebido, fontes de estresse e tipo de ocupação. Os adminstradores mais velhos registravam menor número de fontes 
de estresse, melhor enfrentamento e maior controle interno (Siu, Cooper, Spector, \& Donald, 2001).

Apesar da experiência acumulada, o trabalhador de meia-idade nem sempre é valorizado pelas organizações. De acordo com Neri (2002), frequentemente os trabalhadores mais velhos são excluídos ou discriminados com base nas crenças associadas ao envelhecimento, como doenças, incapacidade cognitiva, isolamento e rigidez social. O crescimento do número de trabalhadores mais velhos tem sido observado na população economicamente ativa (Wajnman, Oliveira, \& Oliveira, 2004). Dados do Instituto Brasileiro de Geografia e Estatística (IBGE, 2006) mostram que os trabalhadores com mais de 50 anos representam 18,1\% da população de trabalhadores das seis regiões metropolitanas, avaliadas mensalmente pela pesquisa de emprego. Esse cenário aponta a necessidade de investimentos em estratégias tanto na manutenção do desenvolvimento da força de trabalho madura quanto na busca de melhores condições para o envelhecimento no trabalho.

\section{Crenças de agência e autoeficácia}

A teoria social cognitiva valoriza as crenças do indivíduo e sua capacidade de atuar como agente, como um importante mediador do comportamento em diferentes processos que envolvem saúde, educação, organizações e trabalho (Bandura, 1977, 1997, 2001, 2006). A agência "envolve não somente a habilidade deliberativa para fazer escolhas e planos de ação mas também a habilidade para dar forma apropriada a um determinado curso de ação e motivar e regular sua execução" (Bandura, 2001, p. 8). A agênca pessoal opera através das crenças de eficácia, definida como as crenças do indivíduo em sua capacidade de organizar e executar determinados cursos de ação para alcançar um resultado, o que representa um recurso crítico para o enfrentamento de estressores (Bandura, 1997). As crenças de agência pessoal e eficácia incluem o exercício de controle através de recursos próprios, tais como o conhecimento, a habilidade e o esforço. Já a autoeficácia coletiva inclui as crenças nos esforços do grupo para agir e para persistir frente às adversidades.

A prevenção e a diminuição do estresse ocupacional requerem que os trabalhadores desenvolvam um forte senso de autoeficácia (Bandura, 1997) e atuem como agentes, capazes de avaliar a capacidade, de agregar julgamento às suas crenças, de antecipar possíveis cursos de ação e resultados e, finalmente, de regular comportamentos em função dos resultados obtidos (Bandura, 2006).

Estudando comportamentos específicos de agência e seu impacto sobre o bem-estar na idade adulta, Smith, Kohn, Savage-Stevens, Finch, Ingate, \& Lim (2000) distinguiram entre crenças de agência pessoal (semelhante ao que Bandura define como controle direto) e crenças de agência interpessoal. As crenças de agência interpessoal incluem comportamentos tais como a comunicação das necessidades e a cooperação com os demais, visando a certos objetivos.

\section{O estudo}

O presente estudo teve como objetivo pesquisar as relações entre idade, experiência de trabalho ( $n$ o de anos trabalhados em determinado cargo em uma companhia) e estratégias de enfrentamento de estressores no trabalho, mediados por crenças de agência e de autoeficácia. Os objetivos específicos foram:

1. identificar os estressores percebidos por 71 trabalhadores adultos que ocupavam posições de liderança em uma empresa de energia elétrica; 
2. avaliar a relação entre as estratégias de enfrentamento e o grau de estresse percebido, a idade, o cargo (técnicos ou engenheiros) e a experiência de trabalho e 3. avaliar o impacto das crenças de agência e de autoeficácia sobre o enfrentamento dos estressores.

\section{Método}

\section{Participantes}

As entrevistas com os trabalhadores e as observações em campo indicaram que os serviços em redes elétricas são potencialmente estressantes. Uma tempestade, por exemplo, pode ser considerada uma ameaça, pois pode implicar desligamento da rede elétrica, sobrecarga de trabalho para restabelecimento de energia e mobilização de recursos, bem como pressões dos clientes. Além disso, o trabalho deve ser executado em postes, envolvendo duplas ou times, exigindo constantes interações, movimentos sincronizados e comportamentos de cooperação e de autocontrole e corpo saudável. Os líderes de tais equipes representam um nível intermediário de gestão, e podem sofrer pressões por parte dos gestores e dos próprios clientes.

O estudo envolveu 34 engenheiros e 37 técnicos de distribuição, o que abrangia 90\% do total de líderes em redes elétricas de uma companhia de energia em São Paulo (a população total de líderes correspondia a 79 pessoas). A idade média foi de 39 anos ( $D P=$ $6,22)$ e variava de 27 a 50 anos. Oito líderes não participaram do estudo, porque não responderam completamente ao protocolo de pesquisa. O tempo médio de experiência foi de cinco anos $(D P=4,75)$, variando de um a 21 anos. Quando se comparam idade e experiência de trabalho, $67 \%$ dos líderes com mais de 40 anos tinham cinco anos ou mais de experiência. Entre os mais jovens, com menos de 40 anos, $45 \%$ tinham de dois a quatro anos de experiência, e 36\%, menos de dois anos, para um p-valor < 0,001 , o que indicou diferenças significativas entre os dois grupos quanto à idade e à experiência de trabalho. Também foram encontradas diferenças significativas em relação à escolaridade: $68 \%$ dos técnicos tinham o segundo grau completo, $56 \%$ dos engenheiros tinham pós-graduação e 44\%, graduação ( $p$-valor < ,001).

O conjunto de dados sociodemográficos que envolvem idade e experiência de trabalho demonstrou tratar-se de população que tende para a meia-idade e que é experiente no trabalho, sugerindo que a liderança de servicos em redes elétricas, nessa companhia, vinha sendo delegada aos mais velhos e mais experientes.

\section{Materiais}

As variáveis pesquisadas foram avaliadas através de: 1. questionário para identificação dos dados sociodemográficos, prática de atividades de lazer e caracterização do trabalho (experiência de trabalho, cargo e se o trabalhador executava atividades fora da companhia); 2. uma questão para avaliar o grau de estresse percebido: Em uma escala de 1 a 10, sendo 10 extremamente estressante e 1 pouco estressante, qual o grau de estresse percebido na execução de serviços em uma empresa de energia?e 3. uma questão aberta para avaliar os estressores ocupacionais: Pense em seu ambiente de trabalho nos últimos doze meses. Identifique o evento ou situação mais estressante que tenha ocorrido nesse período. Os estressores foram agrupados em categorias, segundo a especificidade.

A variável dependente (enfrentamento) foi avaliada através do Inventário de Estresse Ocupacional (Latack, 1986), validado por Pinheiro, Tróccoli e Tamayo (2003), que 
contém 46 itens: 17 itens relativos ao controle, incluindo ações de controle, ações pró-ativas e reavaliação cognitiva, 12 itens relativos à esquiva e à reavaliação cognitiva e 17 itens relativos ao manejo de sintomas. Utilizou-se uma escala tipo Likert, com variação de 1 a 5, da qual constavam os itens: 1 . Nunca faço isso; 2 . Raramente faço isso; 3 . Às vezes faço isso; 4 . Frequentemente faço isso e 5. Sempre faço isso. A validação brasileira mostrou adequada consistência interna, que variou entre:, 77 e ,81.

Para avaliação das crenças de agência, utilizou-se a Escala de Crenças de Agência (Smith et al., 2000), tipo Likert, que variava de um (nunca) a quatro (frequentemente). A escala contém duas dimensões: agência pessoal (8 itens) e agência interpessoal (5 itens), e foi validada semanticamente neste estudo por meio de procedimentos de tradução e retrotradução, realizados por dois professores de língua inglesa, e posterior aplicação a uma amostra de respondentes. $\mathrm{O}$ índice de consistência interna de Cronback foi: ,75 para crenças de agência; ,74 para crenças de agência pessoal e ,44 para crenças de agência interpessoal. Com o objetivo de identificar qual dos itens interferia na consistência interna, realizou-se a análise item a item. O item 2 mostrou-se o item de menor consistência, embora sua exclusão não tenha ocasionado melhora acentuada no índice de Cronback.

As crenças de autoeficácia foram avaliadas através da Escala de Avaliação de Autoeficácia (Jerusalem \& Schwarzer, 1981; Schwarzer \& Costa, 1999), validada por Costa e Pereira (1999), com índice de Cronback, variando entre: ,79 a ,82 em diferentes amostras.

\section{Procedimentos}

A participação foi voluntária, e o protocolo de pequisa foi aplicado por profissionais de recursos humanos da companhia devidamente treinados. Os participantes receberam informações gerais sobre a pesquisa antes de assinarem o formulário de consentimento informado, e a pesquisa foi aprovada pelo Comitê de Ética da Faculdade de Medicina da UNICAMP.

\section{Análise estatística}

As variáveis categóricas foram analisadas por meio dos Testes Qui-quadrado ou Exato de Fisher (para valores esperados menores que 5). Os escores gerais foram comparados por meio do Teste de Mann-Whitney (para 2 categorias) e do Teste de Kruskal-Wallis (para 3 ou mais categorias) devido à ausência de distribuição normal dos escores. As correlações entre as escalas foram calculadas através do coeficiente de correlação de Spearman. O nível de significância adotado para os testes estatísticos foi de $5 \%(p<.05)$. Para avaliar a relação entre as variáveis independentes, as mediadoras e a critério, utilizou-se a análise de regressão linear.

\section{Resultados}

\section{Estressores ocupacionais percebidos}

Baseado na descrição de estressores sugerida por Figueroa et al. (2001) e na metodologia de análise de conteúdo, proposta por Bardin (1995), os estressores organizacionais foram organizados em três categorias: 1 . estressores organizacionais que se referiam a regras, valores, princípios, metas, objetivos e políticas (exemplos: políticas salariais, de movimentação de pessoal, de desligamento de pessoal) e relações institucionais da organização (com sindicatos), quando atuam como desencadeadoras de tensão psicológica; 2. estressores próprios das tarefas relativas à organização e à execução de tarefas, que envolvem aspectos relacionados a prazos, a quantidade de trabalho (sobrecarga), a falta de recursos para execução das tarefas, a novas 
tecnologias e a exigências de qualidade e segurança no trabalho, a desligamentos de rede elétrica e a acidentes de trabalho; 3. estressores relativos à gestão de pessoas, que envolvem processos de seleção de pessoal, avaliação de desempenho, treinamento, motivação, relacionamento interpessoal, conflitos interpessoais e clima organizacional.

Os engenheiros e técnicos líderes identificaram de maneira bastante similar os estressores organizacionais (3 e 2 respectivamente), os estressores próprios da tarefa e aqueles relativos à organização e à execução das tarefas (25 e 21, respectivamente). Os estressores relativos à gestão de pessoas apareceram com maior frequência entre os técnicos líderes (21 e 10, respectivamente), possivelmente porque sejam os responsáveis pelo contato direto com as equipes de eletricistas de rede. Os estressores próprios das tarefas foram os mais citados por ambos os líderes, e envolviam mais frequentemente pressão nos prazos, sobrecarga de trabalho e falta de recursos, provavelmente devido às pressões das gerências e dos clientes.

\section{Grau de estresse percebido, enfrentamento e crenças de agência e de autoeficácia}

O grau médio de estresse percebido na amostra foi relativamente alto: $7(M=7$; $D P=1,72)$, em uma escala de 01 a 10 . Os participantes registraram uso frequente de estratégias de controle $(M=3,73 ; D P=, 49)$, algumas vezes de estratégias de esquiva $(M=2,07 ; D P=, 50)$ e raramente de manejo de sintomas $(M=1,86 ; D P=, 44)$ para enfrentamento do estresse, em uma escala que variava de 1 (nunca faço isso) a 5 (sempre faço isso).

A frequência média para a escala de autoeficácia foi 3,23 (DP=,37), o que significa que o grupo tende a se sentir moderadamente capaz de lidar com as dificuldades do dia a dia, em uma escala que variava de 1 (= não verdadeiro) a 4 (= totalmente verdadeiro). As escalas de crenças de agência pessoal e interpessoal apresentaram escores médios de 3,18 $(D P=, 41)$ e 2,96 $(D P=40)$, respectivamente, sugerindo que, com frequência, os participantes identificaram crenças de controle pessoal e interpessoal sobre como lidar com as circunstâncias importantes da vida, em uma escala que variava de 1 (nunca) a 4 (sempre).

\section{Análise comparativa entre} estresse percebido, estratégias de enfrentamento, autoeficácia, crenças de agência e cargo ocupado no trabalho (engenheiros e técnicos)

Os participantes tiveram seus escores de enfrentamento, autoeficácia e agência comparados segundo os critérios de idade e cargo. Não foram encontradas diferenças significativas entre os escores de grau de estresse percebido, enfrentamento do estresse, autoeficácia e crenças de agência pessoal quando se compararam trabalhadores mais velhos (mais de 40 anos) com os mais jovens (menos de 40 anos). Uma exceção importante foi o escore apresentado na subescala de crenças de agência interpessoal, em que o grupo mais jovem $(<40$ anos) obteve um score significativamente mais alto $(M=3,09 ; D P=, 44)$ em relação ao mais velho ( $>=40$ anos, $M=2,83 ; D P$ $=, 34 ; p$-valor $=, 006)$, indicando que o grupo mais jovem utiliza mais recursos de outras pessoas para lidar com os estressores.

Quanto ao cargo ocupado, observou-se que o grupo de engenheiros pontuou significativamente mais alto em autoeficácia $(M=3,34 ; D P=, 28)$ em relação aos técnicos $(M=3,13 ; D P=, 41 ; p$-valor $=, 045)$, indicando que os engenheiros tendem a se considerar mais autoeficazes. 
Correlações entre grau de estresse percebido, controle, esquiva e manejo de sintomas, autoeficácia e agência e as variáveis idade e experiência de trabalho

Conforme se observa na Tabela 1, não há correlações significativas entre o grau de estresse percebido e as estratégias de controle, esquiva e manejo de sintomas, autoeficácia e crenças de agência. Foram encontradas correlações positivas entre as variáveis experiência de trabalho e autoeficácia: quanto maior o número de anos trabalhado no cargo de líder, maior o senso de autoeficácia $($,28). Correlações negativas foram encontradas entre idade e crenças de agência interpessoal $(-, 33)$, o que significa que, quanto mais velho o líder, menos ele apresenta crenças de agência interpessoal, ou seja, menos recorre a estratégias de controle mediadas por outros.

Tabela 1. Correlações entre o grau de estresse percebido (GEP); estratégias de enfrentamento: controle (C), esquiva (E), manejo de sintomas (MS); autoeficácia (AE); crenças de agência: pessoal (P) e interpessoal (IP) e as variáveis idade e experiência no trabalho.

\begin{tabular}{|c|c|c|c|c|c|c|c|c|}
\hline & & \multirow[b]{2}{*}{ GEP } & \multicolumn{3}{|c|}{ Estratégias de enfrentamento } & \multirow[b]{2}{*}{$\mathrm{AE}$} & \multicolumn{2}{|c|}{$\begin{array}{l}\text { Crenças de } \\
\text { agência }\end{array}$} \\
\hline & & & $\mathrm{C}$ & $\mathrm{E}$ & MS & & $P$ & IP \\
\hline \multirow{3}{*}{ Idade } & r & 0,04 & $-0,17$ & $-0,1$ & $-0,02$ & $-0,02$ & $-0,14$ & $-0,33$ \\
\hline & $\mathrm{p}$ & 0,72 & 0,17 & 0,90 & 0,87 & 0,85 & 0,26 & 0,01 \\
\hline & $\mathrm{n}$ & 67 & 69 & 69 & 69 & 69 & 69 & 69 \\
\hline \multirow{3}{*}{$\begin{array}{l}\text { Experiência } \\
\text { no trabalho }\end{array}$} & r & $-0,07$ & $-0,04$ & $-0,05$ & 0,03 & 0,28 & $-0,01$ & $-0,07$ \\
\hline & p & 0,57 & 0,71 & 0,67 & 0,78 & 0,02 & 0,91 & 0,54 \\
\hline & $\mathrm{n}$ & 69 & 71 & 71 & 71 & 71 & 71 & 71 \\
\hline
\end{tabular}

Nota. $r=$ Coeficiente de correlação de Spearman ; $p=p$ - valor, $n=$ número de participantes

Conforme mostra a Tabela 2, foram encontradas correlações positivas entre as variáveis 1 . manejo de sintomas e controle $(, 24) ; 2$. manejo de sintomas e esquiva $(, 44) ; 3$. autoeficácia e controle: quanto maior a autoeficácia, maior o uso de estratégias de controle $(, 53) ; 4$. crenças de agência pessoal e controle e autoeficácia $(, 67$ e ,56), respectivamente; 5 . crenças de agência interpessoal e controle (,36); 6 . crenças de agência interpessoal e manejo de sintomas (,27); 7. crenças de agência interpessoal e autoeficácia $(, 40)$ e 8 . crenças de agência interpessoal e crenças de agência pessoal $(, 43)$. 
Tabela 2. Correlações entre Grau de Estresse percebido (GEP); Estratégias de Enfrentamento: Controle (C), Esquiva (E), Manejo de Sintomas (MS); Autoeficácia (AE); Crenças de Agência: Pessoal (P) e Interpessoal (IP).

\begin{tabular}{|c|c|c|c|c|c|c|c|c|}
\hline & & \multirow[b]{2}{*}{ GEP } & \multicolumn{3}{|c|}{ Estratégias de enfrentamento } & \multirow[b]{2}{*}{$\mathrm{AE}$} & \multicolumn{2}{|c|}{$\begin{array}{l}\text { Crenças de } \\
\text { agência }\end{array}$} \\
\hline & & & $\mathrm{C}$ & $E$ & MS & & $\mathrm{P}$ & IP \\
\hline \multirow{3}{*}{$\mathrm{C}$} & $r$ & 0,52 & & & & & & \\
\hline & $p$ & 0,67 & & & & & & \\
\hline & $\mathrm{n}$ & 69 & & & & & & \\
\hline \multirow{3}{*}{$E$} & $r$ & 0,04 & 0,15 & & & & & \\
\hline & $p$ & 0,71 & 0,21 & & & & & \\
\hline & $\mathrm{n}$ & 69 & 71 & & & & & \\
\hline \multirow{3}{*}{ MS } & $r$ & 0,00 & 0,24 & 0,44 & & & & \\
\hline & $p$ & 0,96 & 0,04 & 0,00 & & & & \\
\hline & $n$ & 69 & 71 & 71 & & & & \\
\hline \multirow{3}{*}{$\mathrm{AE}$} & $r$ & 0,14 & 0,53 & 0,03 & 0,06 & & & \\
\hline & $p$ & 0,23 & 0,001 & 0,78 & 0,63 & & & \\
\hline & $n$ & 69 & 71 & 71 & 71 & & & \\
\hline \multirow{3}{*}{$P$} & $r$ & $-0,02$ & 0,67 & 0,06 & 0,18 & 0,56 & & \\
\hline & $p$ & 0,84 & 0,00 & 0,58 & 0,12 & 0,00 & & \\
\hline & $\mathrm{n}$ & 69 & 71 & 71 & 71 & 71 & & \\
\hline \multirow{3}{*}{ IP } & $r$ & $-0,12$ & 0,36 & 0,18 & 0,27 & 0,40 & 0,43 & \\
\hline & $p$ & 0,34 & 0,00 & 0,12 & 0,02 & 0,00 & 0,00 & \\
\hline & $\mathrm{n}$ & 69 & 71 & 71 & 71 & 71 & 71 & \\
\hline
\end{tabular}

Nota. ${ }^{*} \mathrm{r}=$ Coeficiente de correlação de Spearman , $\mathrm{p}=\mathrm{p}$ - valor, $\mathrm{n}=$ número de participantes

Análise de regressão linear univariada e multivariada para estratégias de enfrentamento

Foram realizadas análises univariadas ou modelo simples, e, em seguida, a análise multivariada através do modelo múltiplo, utilizando-se o critério Stepwise de seleção de variáveis.

Somente as variáveis autoeficácia, agência pessoal e agência interpessoal foram significativamente relacionadas com a variável critério estratégias de controle. Os líderes com escores mais altos em estratégias de controle tenderam a apresentar escores mais altos em autoeficácia, agência pessoal e interpessoal (vide tabela 3 ). 
Tabela 3. Análise de regressão linear univariada para estratégias de controle.

\begin{tabular}{lcccc}
\hline Variável & Categorias & p-valor & $\mathrm{R}^{2}(\%)$ & Estimativa \\
& & & & (E.P.) \\
\hline Autoeficácia & Variável contínua & $<0,001$ & 27,44 & $1,48(0,44)$ \\
& & & & $0,70(0,14)$ \\
\hline Agência pessoal & Variável contínua & $<0,001$ & 42,62 & $1,27(0,35)$ \\
& & & & $0,78(0,11)$ \\
\hline Agência interpessoal & Variável contínua & 0,002 & 12,74 & $2,45(0,41)$ \\
& & & & $0,43(0,14)$ \\
\hline
\end{tabular}

Nota. R2=coeficiente de determinação (\% de variabilidade da variável resposta explicada pela variável independente); E.P. $=$ erro padrão; estimativa $=$ valores do intercepto $(1 \mathrm{o}$ valor) e do coeficiente angular $(2 \mathrm{o}$ valor) na reta de regressão $\mathrm{Y}=\mathrm{a}+\mathrm{bX}$. Variável dependente: escore de estratégias de controle; $\mathrm{N}=71$ sujeitos

A análise de regressão linear multivariada, usando o critério Stepwise, revelou relação significativa e conjunta dos escores em agência pessoal e em autoeficácia com o escore em estratégias de controle. Líderes com escores mais altos em estratégias de controle tiveram os escores mais altos em agência pessoal e em autoeficácia, conforme indicado na Tabela 4.

Tabela 4. Análise de regressão linear multivariada para estratégias de controle.

\begin{tabular}{llccc}
\hline $\begin{array}{l}\text { Variáveis } \\
\text { Selecionadas }\end{array}$ & Categorias & p-valor & $\mathrm{R}^{2}(\%)$ & $\begin{array}{c}\text { Estimativa } \\
(\text { E.P. })^{+}\end{array}$ \\
\hline Agência pessoal & Variável contínua & $<0,001$ & 44,00 & $0,86(0,41)$ \\
& & & $0,61(0,12)$ \\
\hline Autoeficácia & Variável contínua & 0,038 & 3,72 & -- \\
& & & & $0,30(0,14)$ \\
\hline
\end{tabular}

Nota.R2=coeficiente de determinação parcial; E.P.=erro padrão; estimativa = valores do intercepto (1o valor) e do coeficiente angular (slope) (2o valor) na reta de regressão. Variável dependente: escore de estratégias de controle $(\mathrm{N}=66)$. Critério Stepwise de seleção de variáveis. R2 Total $=47,72 \%$

Nenhuma das variáveis estudadas teve relação significativa com o escore em estratégias de esquiva. 


\section{Discussão}

O presente estudo envolveu uma investigação descritiva do enfrentamento do estresse no trabalho e sua relação com o estresse percebido, o cargo ocupado, a idade e a experiência de trabalho, hipoteticamente mediada pelas crenças de autoeficácia e agência, de 71 líderes de uma empresa de energia.

Os resultados apontaram o valor da experiência de trabalho e das crenças de autoeficácia e agência para o enfrentamento dos estressores do trabalho. Nesse contexto, ainda que o estresse percebido seja alto, os estressores podem ser controlados por líderes que utilizam estratégias de controle, mediadas por crenças de autoeficácia e agência. Quanto mais o líder faz uso de estratégias de controle para lidar com o estresse, mais ele acredita em sua atuação como agente, mais ele se sente autoeficaz. Quanto mais frequentes as crenças de agência pessoal e interpessoal, maior o uso de estratégias de controle para enfrentamento do estresse. A relação entre o enfrentamento e as crenças de controle foi também indiretamente confirmada por não ter sido encontrada correlação significativa entre estratégias de esquiva e tais crenças. Dessa forma, o conceito de enfrentamento parece estar diretamente associado ao exercício do controle na idade adulta, mediado por crenças de controle.

Diferentes tipos de estressores no contexto de trabalho podem predizer diferentes estratégias de enfrentamento. Diante de situações de conflito entre chefes e subordinados e a sobrecarga de trabalho, os indivíduos tendem a fazer uso do enfrentamento ativo, quando se percebe que tais estressores podem ser manejados através de ações (Latack, 1986). No presente estudo, os estressores foram organizados em três categorias: organizacional, relacionados às tarefas ou às pessoas, sendo que os estressores relativos ao manejo de tarefas e a pessoas foram registrados com maior frequência, não tendo sido encontradas, no entanto, diferenças significativas entre as estratégias de enfrentamento de tais estressores. Esses resultados estão de acordo com os de Folkman e Lazarus (1980), sugerindo que a escolha de uma estratégia de enfrentamento tende a ser uma função do meio. Isso inclui a avaliação do quão eficaz alguém se sente para o enfrentamento.

Não foram encontradas relações significativas quando comparadas as estratégias de enfrentamento, a idade e a experiência de trabalho. Levando-se em consideração a idade, esses resultados confirmam estudos anteriores, nos quais se avaliou a influência da idade sobre os mecanismos de enfrentamento, sugerindo que as pessoas mais velhas usam as mesmas estratégias de enfrentamento que as mais jovens (Aldwin, 1991; Aldwin \& Gilmer, 2003; Aldwin et al., 1996). Aldwin e Gilmer (2003) encontraram resultados empirícos contraditórios ao investigar se havia diferenças de enfrentamento associadas à idade. Eles verificaram que os indivíduos mais velhos se consideram menos responsáveis pelos problemas e fazem uso de estratégias centradas no problema tanto quanto os mais jovens, e concluíram que não há diferenças quanto à eficácia percebida do enfrentamento.

A experiência de trabalho foi positivamente correlacionada com autoeficácia, e esta com estratégias de controle, sugerindo que, quanto maior o número de anos trabalhados como líder, maior o senso de que é capaz de produzir resultados, ou seja, de controlar os estressores. Isso sugere que a experiência de trabalho pode estar relacionada a vantagens para o exercício de papéis de liderança em ocupações de risco, e pode também se supor que os líderes que se sentem mais capazes de lidar com os riscos do trabalho são capazes 
de controlar a ansiedade e o estresse. De acordo com a literatura, quanto maior a eficácia percebida, menos os indivíduos se sentem influenciáveis a estímulos disruptivos, acreditando que serão capazes de lidar com as ameaças (Bandura, 1993, 2001).

A liderança de serviços em redes elétricas nessa companhia estava sendo realizada por profissionais próximos da meiaidade e que tinham experiência em suas posições de liderança. Carstensen (1995) e Carstensen, Fung e Charles (2003), ao mostrarem os fundamentos da teoria de seletividade socioemocional, afirmam que os indivíduos mais velhos priorizam a regulação socioemocional, evitando emoções negativas, em detrimento de objetivos cognitivos de expansão e de conquista, característicos de idades mais jovens. A experiência parece desempenhar um importante papel para explicar o aumento da regulação emocional através do curso de vida. Pode-se supor que a regulação emocional seja mais forte em indivíduos mais velhos e em líderes mais experientes no contexto de trabalho. Os líderes mais experientes ainda se apoiam em seus próprios recursos, tal como ficou demonstrado pelos resultados nos quais os líderes com escores mais altos em estratégias de controle tiveram os escores mais altos em agência pessoal, ou seja, utilizam mais estratégias ativas para enfrentamento, baseando-se em recursos próprios. Já os líderes mais jovens utilizam mais recursos do outro para lidar com os estressores (agência interpessoal) em relação aos mais velhos.

Possivelmente, este estudo pode ser generalizado somente para companhias com características similiares, considerandose a estrutura das carreiras e as pressões naturalmente inerentes ao exercício do trabalho em companhias de energia elétrica. Além disso, somente foram utilizadas medidas quantitativas. A inclusão de medidas qualitativas, como entrevistas e diários, poderia melhor caracterizar os estressores do trabalho associados a riscos de vida, característicos do trabalho em redes de energia.

Outra limitação do estudo é que foram investigadas somente as crenças pessoais de controle (agência pessoal, interpessoal e autoeficácia). As crenças de agência coletiva não foram avaliadas, e, de acordo com a teoria, altos níveis de autoeficácia coletiva percebida geram altas aspirações, motivação e persistência frente às dificuldades, maior resiliência moral a estressores e alta performance (Bandura, 1997).

\section{Conclusões}

Os resultados deste estudo apontaram o valor das crenças de agência e autoeficácia para o efetivo enfrentamento de estressores, principalmente para uma população que envelhece no ambiente de tabalho, executando serviços sujeitos a risco de vida. O enfrentamento de estressores pode ser um processo muito mais relacionado a sentir-se capaz de lidar com os estressores e a atuar como um agente diante das condições adversas do que como um processo dependente da idade ou do uso de determinada estratégia. A experiência de trabalho pode contribuir para o enfrentamento de estressores, especialmente em redes elétricas.

Os resultados encontrados permitem o questionamento de conceitos preestabelecidos relativos ao declínio em competências cognitivas e sociais de trabalhadores mais velhos, e sugere que as organizações deveriam valorizar a mão de obra madura. Essas organizações poderiam priorizar, em seus programas de treinamento, o desenvolvimento de habilidades pró-ativas de enfrentamento, tais como as habilidades 
de autorregulação (automonitoramento, avaliação de autoeficácia, estabelecimento de objetivos e autoincentivos específicos), de análise e resolução de problemas, de tomada de decisões e as competências socioemocionais (empatia, comunicação, manejo de confitos e trabalho em equipes).

Estudos que envolvessem trabalhadores mais velhos do que os incluídos nesta amostra permitiriam melhor compreensão das variáveis relacionadas à experiência de trabalho, especificamente à autoeficácia, à agência e ao enfrentamento, o que possibilitaria a adoção de medidas preventivas que pudessem aumentar a saúde mental de trabalhadores idosos.

Arlete Portella Fontes*

Mestre pelo Programa de Gerontologia, Faculdade de Educação, Universidade Estadual de Campinas, São Paulo - SP - Brasil.

\section{Anita Liberalesso Neri}

Livre Docente em Educação, Professora Titular no Programa de Gerontologa, Faculdade de Ciências Médicas, Universidade Estadual de Campinas- SP- Brasil

E-mail: anitalbn@uol.com.br

\section{Mônica Sanches Yassuda}

Professora Dra. no Curso de Bacharelado em Gerontologia da Escola das Artes, Ciências e Humanidades, Universidade de São Paulo - SP - Brasil.

E-mail: yassuda@usp.br

\section{*Endereço para envio de correspondência:}

Rua General Marcondes Salgado, 769, ap. 71 Campinas, São Paulo - SP - Brasil. CEP 13026-075.

E-mail: arletepfontes@gmail.com 
Referências
Aldwin, C. M. (1991). Does age affect the stress and coping process? Implications of age differences in perceived control. Journal of Gerontology: Psychological Sciences, 46(4), 174180.

Aldwin, C. M., \& Gilmer, D. F. (2003). Health, illness, and optimal aging: Biological and psychosocial perspectives. Thousand Oaks, CA: Sage.

Aldwin, C. M., Sutton, K. J., Chiara, G., \& Spiro III, A. (1996). Age differences in stress, coping, and appraisal: Findings from the normative aging study. Journal of Gerontology Series B: Psychological Sciences and Social Sciences, 51(4), 179-188.

Bandura, A. (1977). Self-efficacy: Toward a unifying theory of behavioral change. Psychological Review, 84(2), 191-215.

Bandura, A. (1993). Perceived self-efficacy in cognitive development and functioning. Educational Psychologist, 28, 117-148

Bandura, A. (1997). Self - efficacy: The exercise of control. New York: W. H. Freeman.

Bandura, A. (2001). Social cognitive theory: An agentic perspective. Annual Reviews Psychology, 2, 1-26.

Bandura, A. (2006). Towards a psychology of human agency. Perspectives on Psychological Science, 1(2), 164-180.

Bardin, L. (1995). Análise do conteúdo (L. A. Reto \& A. Pinheiro, trad.). Lisboa: Edições 70

Carstensen, L. L. (1995). Motivação para contato social ao longo do curso de vida: uma teoria de seletividade socioemocional. In A. L. Neri (Org.), Psicologia do envelhecimento: temas selecionados na perspectiva de curso de vida (pp. 111-144). Campinas, SP: Papirus.

Carstensen, L. L., Fung, H. H., \& Charles, S. T. (2003). Socioemotional selectivity theory and the regulation of emotion in the second half of life. Motivation and Emotion, $27(2), 103-123$

Costa, B., \& Pereira, Y. (1999). Validando uma escala de autoeficácia geral. In Anais da Sociedade Brasileira para o Progresso da Ciência, BRA, 51. Porto Alegre: SBPC.

Figueroa, N. L., Schufer, M., Muiños, R., Marro, C., \& Coria, E. A. (2001). Um instrumento para a avaliação de estressores psicossociais no contexto de emprego. Psicologia: Reflexão e Crítica, 14(3), 653-659.

Folkman, S., \& Lazarus, R. S. (1980). An analysis of doping in a middle-age community sample. Journal of Health and Social Behavior, 21, 219-239.

Folkman, S., \& Mosckowitz, J. T. (2000). Positive affect and the other side of coping. American Psychologist, 55(6), 647-654.

Instituto Brasileiro de Geografia e Estatística. (2006). Pesquisa Mensal de Emprego. Recuperado em março de 2006, de http://www.ibge.gov.br/home/presidencia/noticias/noticia visualiza.php?id noticia $=648 \&$ id pagina $=1$

Jerusalem, M., \& Schwarzer, R. (2004). The general self-efficacy scale (GSE). Recuperado em 23 de maio de 2004, de http:// userpage.fu-berlin.de/ health/engscal.htm

Jex, S. M., Bliese, P. D., Buzzell, S., \& Primeau, J. (2001). The impact of self-efficacy on stressor-strain relations: Coping style as an explanatory mechanism. Journal of Applied Psychology, 86(3), 401-409.
Latack, J. C. (1986). Coping with job stress: Measures and future directions for scale development. Journal of Applied Psychology, 71(3), 377-385.

Lazarus, R. S. (1993). From psychological stress to the emotions: A history of changing outlooks. Annual Review of Psychology, $44,1-21$

Lazarus, R. S., \& Folkman, S. (1984). Stress appraisal and coping. New York: Springer.

Levi, L. (1998). Factores psicosociales, estrés y salud. In Organización Internacional del Trabajo, Enciclopedia de salud y seguridad en el trabajo: Vol. 2. Factores psicosociales y de organización (pp. 34.3-34.6). Madrid: OIT

Michie, S., \& Williams, S. (2003). Reducing work related psychological ill health and sickness absence: A systematic literature review. Occupational Environment Medicine, 60, 3-9.

Neri, A. L. (2002). Envelhecer bem no trabalho: possibilidades individuais, organizacionais e sociais. Terceira Idade, 13(24), 7-27.

Pinheiro, F. A., Tróccoli, B. T., \& Tamayo, M. R. (2003). Mensuração de coping no ambiente ocupacional. Psicologia: Teoria e Pesquisa, 19(2), 153-158.

Schwarzer, R., \& Costa E. B. (1999). Autoeficácia percebida. Recuperado em 26 de maio de 2004 , de http://userpage. fu-berlin.de/ health/htm

Siu, O., Cooper, C. L., Spector, P. E., \& Donald, I. (2001). Age differences in coping and locus of control: A study of managerial stress in Hong Kong. Psychological Aging, 16(4), 707-710.

Smith, G. C., Kohn, S. J., Savage-Stevens, S. E., Finch, J. J., Ingate, R., \& Lim Y. (2000). The effects of interpersonal and personal agency on perceived control and psychological well-being in adulthood. The Gerontologist, 40, 458-468.

Wajnman, S., Oliveira, A. M. H. C., \& Oliveira, E. L. (2004). Os idosos no mercado de trabalho: tendências e consequências. In A. A. Camarano (Org.), Os novos idosos brasileiros: muito além dos 60? (pp. 453-479). Rio de Janeiro: IPEA. 